\author{
Military Technical College \\ Kobry El-Kobbah, \\ Cairo, Egypt
}

\author{
$6^{\text {th }}$ International Conference \\ on Electrical Engineering \\ ICEENG 2008
}

\title{
A proposed approach for online transient stability Of multimachine power systems using parallel microprocessors
}

\author{
By
}

M. A. Ali* and W. M. Mansour**

\section{Abstract:}

Real time transient stability analysis is a challenging computing problem. In order to speed up the solution of this problem, parallel processing technologies have been applied. In this paper, the implementation of parallel algorithms for power system transient stability analysis (TSA) on parallel microprocessors is described. Test simulations are performed for a large-scale power system using MATLAB/SIMULINK model. Results for both proposed method and conventional method are presented to show the identification of the proposed algorithms.

\section{Keywords:}

Parallel processing, parallel algorithms, online simulation, and transient stability analysis.

\footnotetext{
* Graduate student, Dpt. of Electrical Engineering, Benha University, Cairo, Egypt.

** Professor, Dpt. of Electrical Engineering, Benha University, Cairo, Egypt.
} 


\section{Introduction:}

An important problem in power systems is stability. Many major recent blackouts, caused by power system instability, show the importance of this phenomenon. Transient stability has been the dominant stability problem on most systems, and has been the focus of much of the industry's attention concerning system stability assessment. Transient stability assessment is concerned with the behavior of the synchronous machines after they have been perturbed following a large disturbance in a system. A variety of transient stability assessment methods which including time domain solutions, extended equal area criteria, and direct stability methods such as transient energy function are discussed in $[2,3,5,6,10]$.

In the time domain simulation method, the initial system state is obtained from the prefault system. This is the starting point used for the integration of the fault-on dynamic equations. After the fault is cleared, the post-fault dynamic equations are numerically integrated. The machine angles may be plotted versus time and analyzed. If these angles are bounded, the system is stable, otherwise it is unstable.

On the one hand, time domain simulation provides the most accurate approach for determining the transient stability of the system. This method allows flexible modeling of components. However, it is computationally intensive and not suitable for online application. On the other hand, a direct method in TSA offers the opportunity of assessing the transient stability of power systems without explicitly solving differential equations. Therefore, they are computationally fast and suitable for real-time transient stability analysis. However, they require significant approximations which limit modeling accuracy [1].

In this paper another method for online transient stability assessment of large scale power systems is proposed. The method is based on an exact mathematical transformation of an n-machine system into n-equivalent single machine-to equivalent bus (SMEB) system equations. Then implementing the derived parallel algorithms for power system TSA on parallel microprocessors using MATLAB/SIMULINK model for system simulation $[4,11]$. Thus a considerable reduction in computational simulation time is obtained and approaching the online simulation [6-9,11,12,17].

\section{Proposed Method Algorithms:}

A large-scale multimachine electrical power system is considered to derive a detailed mathematical model for each machine in the system to build an equivalent model which is denoted by single machine-to equivalent bus SMEB. A single line diagram depicts the network configuration for machine-i is shown in fig.(1). 


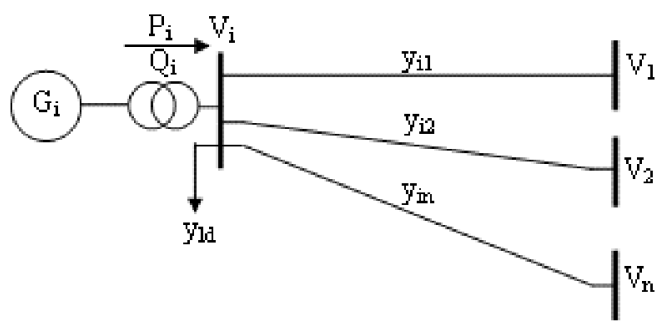

Figure (1): A group of buses are connected to synchronous machine via an overhead transmission line for each bus. Where buses 1,2 , and $\mathrm{n}$ are connected to other system elements

Firstly, determining the injected current at machine bus-i from the original configuration of the system,

$$
\tilde{\mathrm{I}}_{\mathrm{i}}=\frac{\tilde{S}_{i}}{\tilde{V}_{i}^{*}}=\sum_{\substack{J=1 \\ J \neq i}}^{n}\left(\tilde{\mathrm{V}}_{\mathrm{i}}-\tilde{\mathrm{V}}_{\mathrm{j}}\right) \tilde{\mathrm{Y}}_{\mathrm{ij}}+\tilde{\mathrm{V}}_{\mathrm{i}} \tilde{\mathrm{Y}}_{\mathrm{pi}}
$$

Where,

$\tilde{\mathrm{V}}_{\mathrm{i}}=\mathrm{V}_{\mathrm{i}} \mathrm{e}^{\mathrm{j} \alpha_{\mathrm{i}}} \quad ; \tilde{\mathrm{V}}_{\mathrm{j}}=\mathrm{V}_{\mathrm{j}} \mathrm{e}^{\mathrm{j} \alpha_{\mathrm{j}}} \quad ; \tilde{\mathrm{Y}}_{\mathrm{ij}}=\mathrm{Y}_{\mathrm{ij}} \mathrm{e}^{\mathrm{j} \mathrm{p}_{\mathrm{ij}}} \quad ; \tilde{S}_{i}=P_{i}+j Q_{i} \quad ; \tilde{\mathrm{Y}}_{\mathrm{pi}}=\tilde{\mathrm{Y}}_{\text {shi }}+\tilde{\mathrm{Y}}_{\mathrm{ldi}}$

After rearranging the previous equation the following equations will be derived.

$$
\begin{aligned}
\frac{P_{i}}{V_{i}} & =V_{i} Y_{i i} \cos \rho_{i i}-\sum_{\substack{j=1 \\
j \neq i}}^{n} V_{j} Y_{i j} \cos \left(\rho_{i j}-\alpha_{i}+\alpha_{j}\right) \\
& =V_{i} B_{i}-D_{i} \cos \alpha_{i}-C_{i} \sin \alpha_{i}
\end{aligned}
$$

Also,

$$
\begin{aligned}
\frac{Q_{i}}{V_{i}} & =V_{i} Y_{i i} \sin \rho_{i i}-\sum_{\substack{j=1 \\
j \neq i}}^{n} V_{j} Y_{i j} \sin \left(\rho_{i j}-\alpha_{i}+\alpha_{j}\right) \\
& =V_{i} A_{i}-C_{i} \cos \alpha_{i}+D_{i} \sin \alpha_{i}
\end{aligned}
$$


Where,
$A_{i}=Y_{i i} \sin \rho_{i i}$
; $B_{i}=Y_{i i} \cos \rho_{i i}$
$C_{i}=\sum_{\substack{j=1 \\ j \neq i}}^{n} V_{j} Y_{i j} \sin \left(\rho_{i j}+\alpha_{j}\right)$
$; D_{i}=\sum_{\substack{j=1 \\ j \neq i}}^{n} V_{j} Y_{i j} \cos \left(\rho_{i j}+\alpha_{j}\right)$

From the equivalent SMEB shown in fig.(2) another formula could be obtained for the injected current at bus-i.

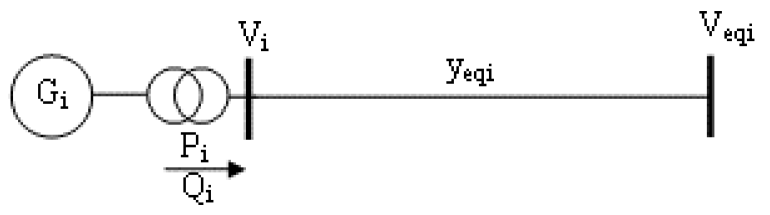

Figure (2): Single machine-to equivalent bus SMEB

Secondly, determining the injected current at machine bus-i from the equivalent SMEB system,

$\tilde{\mathrm{I}}_{\mathrm{i}}=\frac{\tilde{S}_{i}}{\tilde{V}_{i}^{*}}=\left(\tilde{\mathrm{V}}_{\mathrm{i}}-\tilde{\mathrm{V}}_{\text {eqi }}\right) \tilde{\mathrm{Y}}_{\text {eqi }}$

Where,

$\tilde{\mathrm{V}}_{\text {eqi }}=\mathrm{V}_{\text {eqi }} \mathrm{e}^{\mathrm{j} \alpha_{\text {eqi }}} ; \tilde{\mathrm{Y}}_{\text {eqi }}=\mathrm{Y}_{\text {eqi }} \mathrm{e}^{\mathrm{j} \mathrm{j}_{\text {eqi }}}$

After some calculations the following equations will be obtained.

$\frac{P_{i}}{V_{i}}=V_{i} Y_{e q i} \cos \rho_{e q i}-V_{e q i} Y_{e q i} \cos \left(\rho_{e q i}-\alpha_{i}+\alpha_{e q i}\right)$

Also,

$\frac{Q_{i}}{V_{i}}=V_{i} Y_{e q i} \sin \rho_{e q i}-V_{e q i} Y_{e q i} \sin \left(\rho_{e q i}-\alpha_{i}+\alpha_{e q i}\right)$

Comparing equations $(2,3)$ with equations $(5,6)$ respectively, the equivalent parameters of the SMEB can be obtained. 


$$
\left.\begin{array}{ll}
Y_{e q i}=\sqrt{A_{i}^{2}+B_{i}^{2}} & ; \rho_{e q i}=\operatorname{atan}\left(\frac{A_{i}}{B_{i}}\right) \\
V_{e q i}=\frac{\sqrt{C_{i}^{2}+D_{i}^{2}}}{Y_{e q i}} & ; \alpha_{e q i}=\operatorname{atan}\left(\frac{C_{i}}{D_{i}}\right)-\rho_{e q i}
\end{array}\right\}
$$

\section{Adaptation Of Parallel Microprocessors:}

Using the proposed approach the system is converted to groups of SMEB. Thus the adaptation of parallel microprocessors is suitable using the proposed parallelized algorithms [13-16]. The system comprises central microprocessor (CMP) and decentralized microprocessors (DCMP) for each machine. The CMP receives the output variables from each machine microprocessor DCMP then CMP executes its function which is transferring the required variables to each machine DCMP which will be used to compute the updated equivalent parameters of the equivalent models of the machines. Schematic diagram to represent the adaptation of parallel microprocessors using the parallelized algorithms is shown in fig.(3).

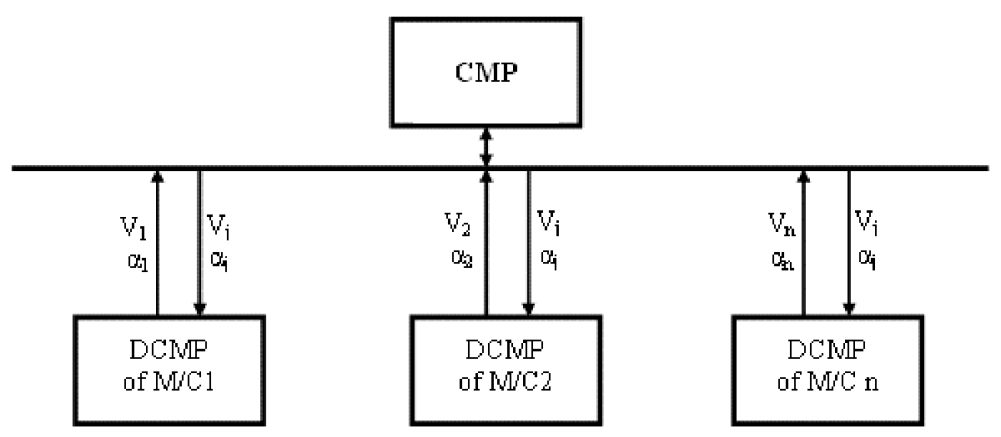

Figure (3): Schematic diagram representing the parallelized algorithms

\section{Case Under Study:}

The proposed approach is adapted on 9-bus, 3-machine test system used in [2], with bus1 is taken as an infinite bus. System configuration is shown in fig.(4). Then the test system is reduced to two groups of SMEB using the proposed approach as shown in fig.(5). 


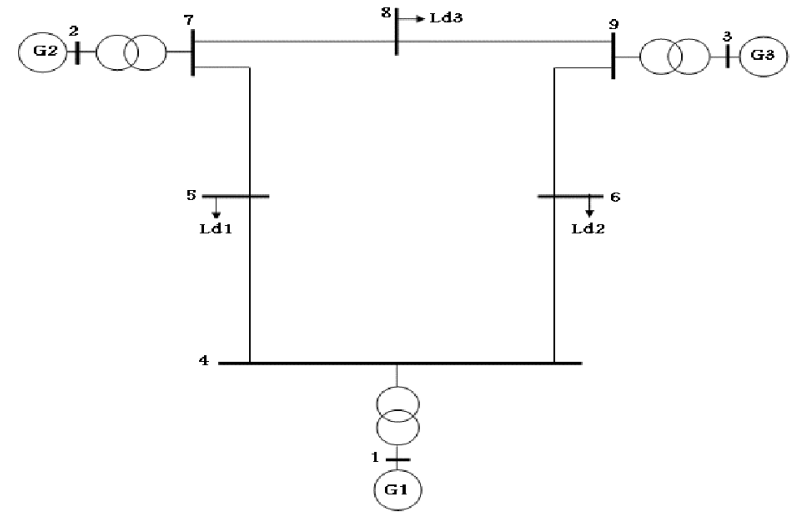

Figure (4): Network configuration of the test system
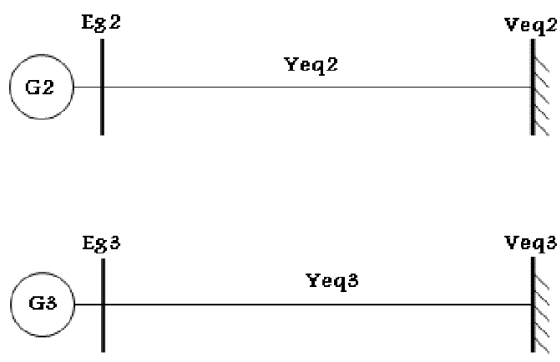

Figure (5): Two groups of SMEB produced by the proposed approach

The equivalent parameters are evaluated according to the derived equations in (7), and then a MATLAB/SIMULINK model based on MATLAB 7.0 is constructed to simulate the power system when subjected to various physical disturbances.

\subsection{System Results:}

A MATLAB/SIMULINK model shown in fig.(6.a) is constructed based on the proposed method using the classical model for synchronous machine representation to obtain rotor angle $(\delta)$, angular speed $(\omega)$ of the two machines. When the system is subjected to; Firstly, sustained $20 \%$ sudden reduction in mechanical torque of machine3, System results are shown in fig.(7). Secondly, 300ms three phase short circuit at the infinite bus as shown in fig.(8).

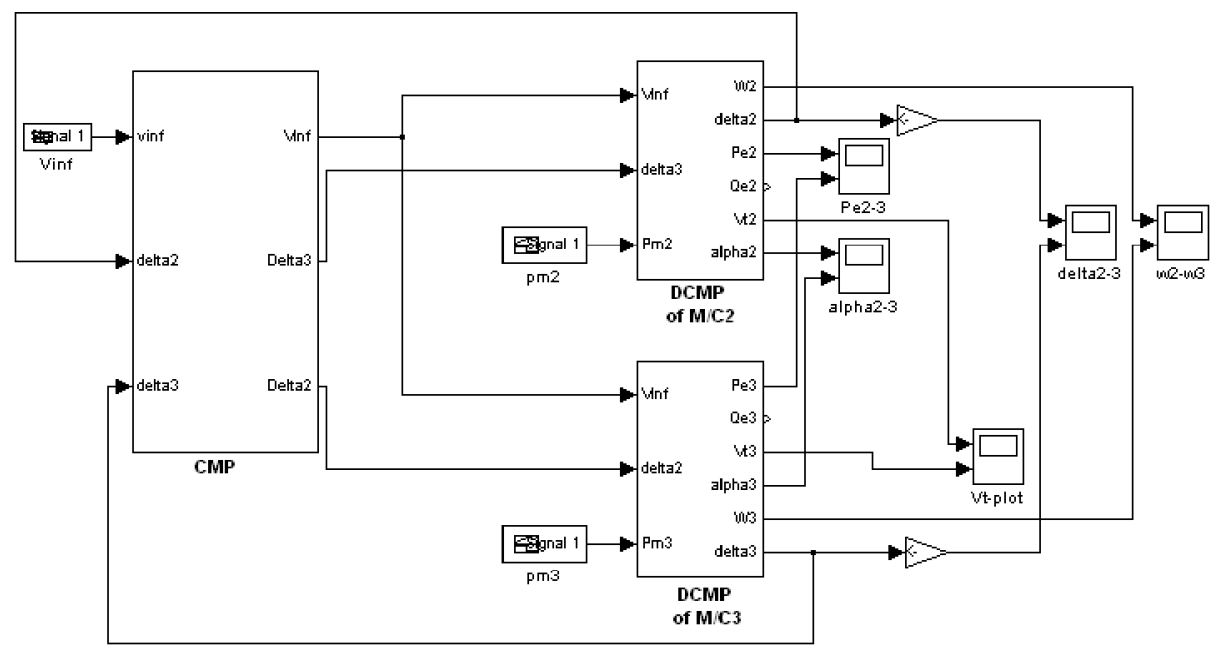

Figure (6):a. MATLAB/SIMULINK model representing the operation of parallel microprocessors 
Also, MATLAB/SIMULINK model shown in fig.(6.b) is constructed based on conventional method using the classical model for synchronous machine representation to obtain the same parameters and identical time responses are given as shown in fig. $(7,8)$.

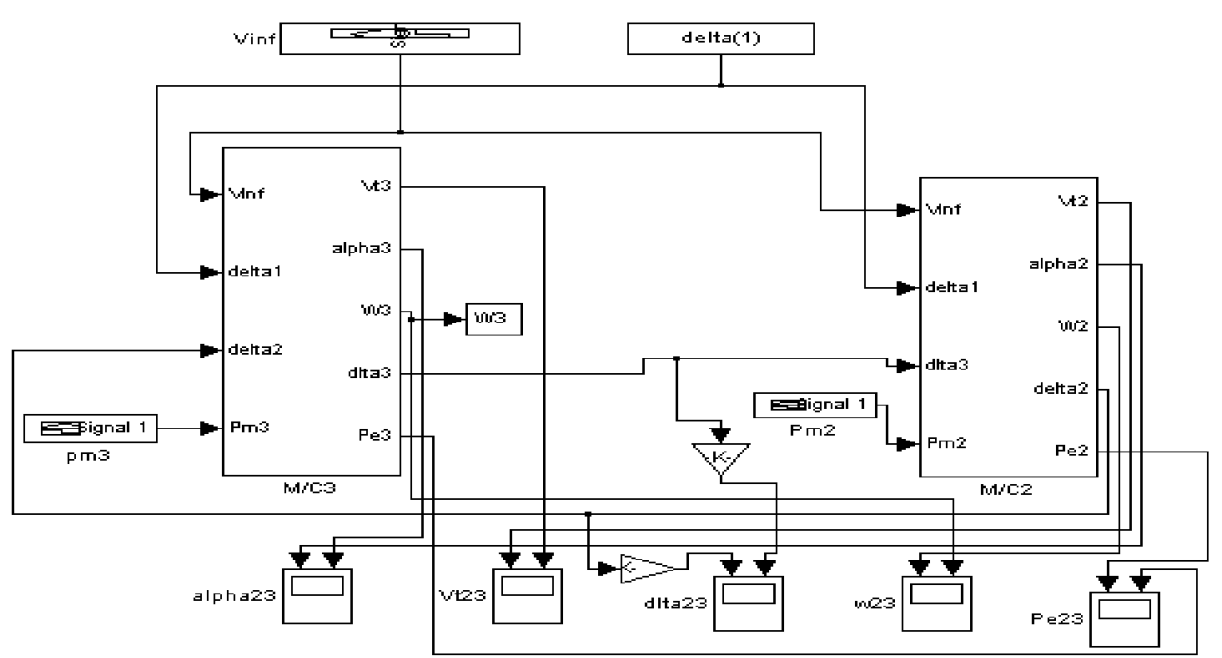

Figure (6):b. MATLAB/SIMULINK model using conventional method
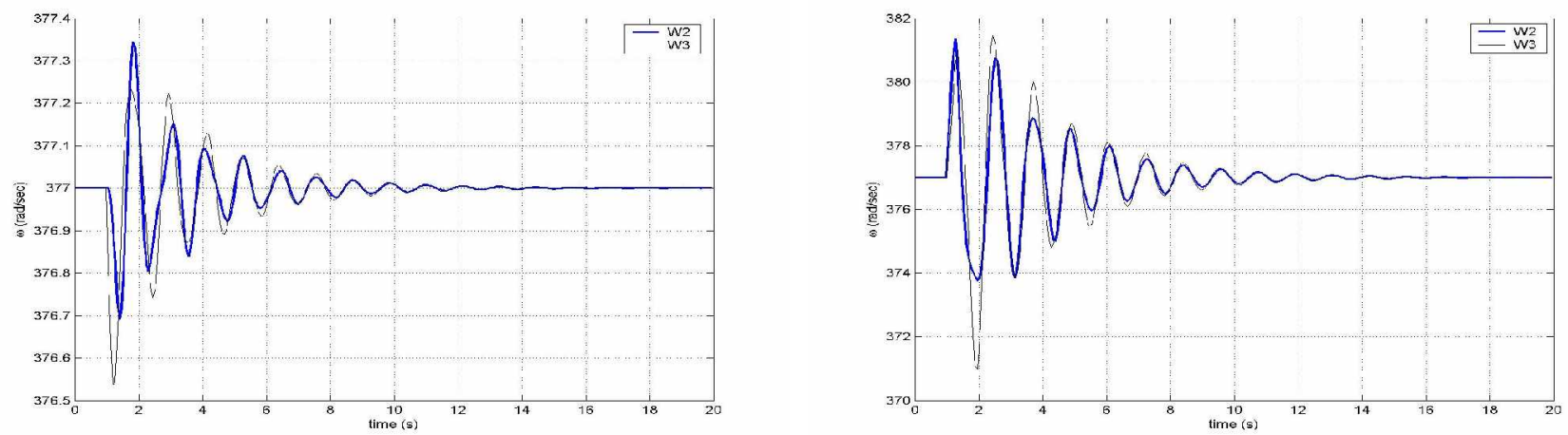

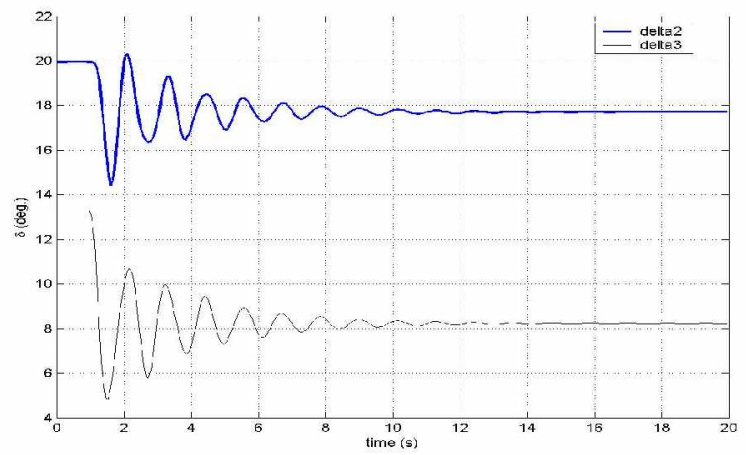

Figure (7): $\omega_{2}, \omega_{3}, \delta_{2}$ and $\delta_{3}$ due to sustained $20 \%$ sudden reduction in mechanical torque of machine 3

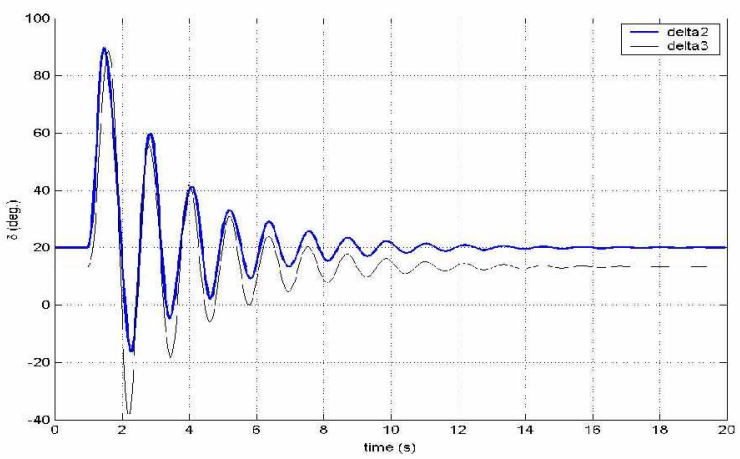

Figure (8): $\omega_{2}, \omega_{3}, \delta_{2}$ and $\delta_{3}$ due to $300 \mathrm{~ms}$ three phase short circuit at the infinite bus 


\section{Conclusions:}

In this paper a detailed mathematical model for parallel algorithms is derived to solve the TSA without using any iterative method so that, a considerable reduction in computational simulation time is obtained. Also a MATLAB/SIMULIK model is constructed based on the conventional time domain method and identical responses due to various physical disturbances are obtained. So that this method has a wide range of applications in online control, adaptive control, and transient stability analysis calculations especially for large-scale power systems.

\section{References:}

[1] Wieslaw T. Kwasnicki; "High Speed Transient Stability Multiprocessing Solutions", PhD., The University of Manitoba Wuinipeg, Manitoba, Canada, June 1998

[2] P. M. Anderson and A. A. Fouad; "Power system and control", volume1.

[3] Olle L. Elgerd ; "Electric energy systems theory", second edition

[4] Ramnarayan Patel, T. S. Bhatti, D. P. Kothari; "MATLAB/Simulink based transient stability analysis of a multimachine power system"; International Journal of Electrical Engineering Education ISSN: 0020-7209 Volume 39 Issue 4, October 2002, pp 320-336.

[5] Xue, Y.Van Cutsem, T.Ribbens-Pavella. M.; "A simple direct method for fast transient stability assessment of large power systems"; Power Systems, IEEE Transactions on Publication Date: May 1988 Volume: 3, Issue: 2, pp: 400-412 ISSN: 0885-8950

[6] Rahimi, A. Systems, Man and Cybernetics; "Generalized equal-area criterion: a method for on-line transient stability analysis", Conference Proceedings., IEEE International Conference on Volume, Issue, 4-7 Nov 1990, pp: 684 - 688.

[7] Yoon-Sung Cho, Jungsoo Park and Gilsoo Jang; "A novel tool for transient stability analysis of large-scale power systems", ScienceDirect, Simulation Modelling Practice and Theory, Volume 15, Issue 7, August 2007, pp: 786-800.

[8] Fugii Wataru, and Iwamoto Shin'Ichi; "Transient Stability Analysis based on Dynamic Single Machine Equivalent", Papers of Technical Meeting on Power Engineering, IEE Japan, Volume PE-06;NO.13-16.18-23, pp:13-18(2006).

[9] Fujii, Wataru Wakisaka, Jun Iwamoto, and Shinichi; "Transient Stability Analysis Based on Dynamic Single Machine Equivalent", Power Symposium, 2007. NAPS '07. 39th North American, Oct.,2-2007, pp: 217-222.

[10] Glenn W. Stagg, and Ahmed H. El-Abiad; "Computer Methods in Power Systems Analysis". 
[11] Bhat, S, Glavic, M, Pavella, M, Bhatti, T S, Kothari, D P ; "A transient stability tool combining the SIME method with MATLAB and SIMULINK", International Journal of Electrical Engineering Education, Apr. 2006.

[12] Young-Hyun Moon; Byjng-Hoon Cho; Byung-Kon Choi; "Fast time simulation technique using noniterative algorithm of transient stability analysis", Power Engineering Society Winter Meeting, 2000. IEEE Volume 1, Issue , 2000, pp:52 - 57 vol.1.

[13] Machado Ferreira, C.; Beleza Carvalho, J.; Dias Pinto, J.A.; Maciel Barbosa, F.P.; "On-line transient stability assessment of an electric power system using a parallel processing state estimator", Electrotechnical Conference, 2000. MELECON 2000. 10th Mediterranean Volume 3, Issue , 29-31 May 2000, pp: 1120 - 1123 vol.3.

[14] Hong, C.; Shen, C.M.; "Implementation of parallel algorithms for transient stability analysis on a message passing multicomputer", Power Engineering Society Winter Meeting, 2000. IEEE Volume 2, Issue, 2000, pp: 1410 - 1415.

[15] Aloisio, G.; Bochicchio, M.A.; La Scala, M.; Sbrizzai, R.; "A distributed computing approach for real-time transient stability analysis", Power Systems, IEEE Transactions on volume 12, Issue 2, May 1997, pp: 981 - 987.

[16] Wang, F.Z. Generation; "Parallel-in-time relaxed Newton method for transient stability analysis", Transmission and Distribution, IEE Proceedings- Volume 145, Issue 2, Mar 1998, pp:155 - 159.

[17] Fang Da-Zhong; Chung, T.S.; David, A.K.; "Fast transient stability estimation using a novel dynamic equivalent reduction technique", Power Systems, IEEE Transactions on Volume 9, Issue 2, May 1994, pp: 995 - 1001.

\section{Nomenclatures:}

$\delta \ldots$ Power angle

$\omega \ldots$ Rotor angular speed

$\alpha_{i}, \alpha_{j} \ldots$ Voltage angle of bus-i, and bus- $j$ respectively

$\rho_{\mathrm{ij}} \ldots$ Angle of line admittance between bus- $i$ and bus- $j$

$\rho_{\mathrm{ii}} \ldots$ Angle of self admittance of bus-i from the bus admittance matrix

$\alpha_{\text {eqi }} \ldots$ Voltage angle of the equivalent artificial bus connected to machine-i

$\rho_{\text {eqi }} \ldots$ Angle of line admittance connected to machine- $i$

$\tilde{\mathrm{I}}_{\mathrm{i}} \ldots$ Injected current at bus-i

$\mathrm{P}_{\mathrm{i}} \ldots$ Injected active power at bus-i

$\mathrm{Q}_{\mathrm{i}} \ldots$ Injected reactive power at bus-i

$\tilde{S}_{i} \ldots$ Injected apparent power at bus-i

$\tilde{V}_{i}, \tilde{V}_{j} \ldots$ Voltage of bus-i, and bus-j respectively 
$\tilde{\mathrm{Y}}_{\mathrm{ij}} \ldots$ Line admittance between bus-i and bus-j

$\tilde{Y}_{i i} \ldots$ The self admittance of bus-i from the bus admittance matrix

$\tilde{Y}_{p i} \ldots$ The summation of line shunt admittance and local load admittance at bus-i

$\tilde{Y}_{\text {shi }} \ldots$ Line shunt admittance at bus-i

$\tilde{Y}_{\text {ldi }} \ldots$ Local load admittance at bus-i

$\mathrm{V}_{\mathrm{i}}, \mathrm{V}_{\mathrm{j}} \ldots$ Voltage magnitude of bus-i, and bus-j respectively

$\mathrm{Y}_{\mathrm{ij}} \ldots$... Magnitude of line admittance between bus-i and bus- $\mathrm{j}$

$\mathrm{Y}_{\mathrm{ii}}$... Magnitude of self admittance of bus-i from the bus admittance matrix

$\tilde{\mathrm{V}}_{\text {eqi }} \ldots$ Voltage of the equivalent artificial bus connected to machine-i

$\mathrm{V}_{\text {eqi }} \ldots$ Voltage magnitude of the equivalent artificial bus connected to machine- $\mathrm{i}$ 